\title{
New Approach to the Measurement of the Specific Heat of Gases*
}

\author{
S. C. Coluins, Cryogenic Laboratory, Massachusetts Institute of Technology, Cambridge, Massachusetts \\ AND \\ G. J. Van Wylen, Mechanical Engineering Department, University of Michigan, Ann Arbor, Michigan
} (Received December 3, 1954)

\begin{abstract}
The variation of temperature with elevation within a body of gas in convective equilibrium in a gravitational field depends upon the specific heat. Specifically, the sum of the enthalpy and potential energy is the same for all elevations. It is only necessary, therefore, to measure the temperature at two points and the vertical distance between them to find the specific heat at the existing pressure. A calorimeter for securing an approximation to convective equilibrium is described.
\end{abstract}

$\mathrm{R}^{\mathrm{n}}$ ECENT efforts to develop a new method of measuring the specific heat of gases have shown some promise. In view of the fact that it has been impossible to continue the project, this report summarizes the work which has been completed.

This method utilizes what might be termed an adiabatic calorimeter, and can be described with the aid of Fig. 1. A long cylindrical pressure vessel is fitted with a concentrically mounted tube and a circulating fan. A charge of experimental gas is forced to flow upward through the annular channel and downward through the tube. If the gas flows adiabatically down the tube and changes in kinetic energy are negligible, the enthalpy of the gas is increased by an amount equal to the decrease in potential energy, as indicated by the steady-flow energy equation

$$
d h+d z=0 .
$$

For a pure substance

$$
\begin{aligned}
T & =f(p, h), \\
d T & =(\gamma T / \gamma P)_{h} d p+(\gamma T / \gamma h)_{p} d h, \\
d T & =\mu d p+1 / c_{p} d h .
\end{aligned}
$$

It may be shown that the first term is negligible as compared to the second and, therefore, substituting Eq. (1) into Eq. (2), we find

$$
c_{p} d T=-d z \text {. }
$$

Integrating between state (1) and state (2), we find

$$
c_{p}=\left(z_{1}-z_{2}\right) /\left(T_{2}-T_{1}\right) \text {. }
$$

This means that by measuring the temperature difference between two known elevations, the specific heat is readily calculated.

One purpose in pursuing such a method is that it seemed suited to measurements at high pressures and low temperatures, requiring simply the charging of the calorimeter to the desired pressure and means for cooling the entire calorimeter.

In the calorimeter which was constructed, the

* This work was supported by a grant from the Aero Medical Laboratory, Dayton, Ohio. pressure vessel was 10 feet long and 14 inches in diameter. The center tube consisted of two concentric sheet metal tubes, 8 in. and $10 \mathrm{in}$. in diameter, with the one-inch annulus between filled with Santocel insulation. A platinum resistance thermometer which consisted of 25 feet of 0.004-in.-diameter platinum wire spiralled on a mica frame, was used in conjunction with a Wheatstone bridge to measure the temperature differences. The thermometer was raised and lowered to the temperature measuring stations and changes in temperature of $0.0015^{\circ} \mathrm{F}$ could be detected.

The most difficult aspect encountered in the experimental work was to maintain so nearly adiabatic flow that temperature changes of the gas resulting from heat transfer were negligible compared to those resulting from the changes in potential energy. Three steps were taken to achieve this.

1. The temperature of the gas in the calorimeter tended to change due to the energy input of the fan and also as a result of heat transfer due to a temperature difference between the room and the calorimeter. The calorimeter was wrapped with a heating coil and four inches of glass wool insulation. The calorimeter was

FIG. 1. Schematic diagram of adiabatic colorimeter

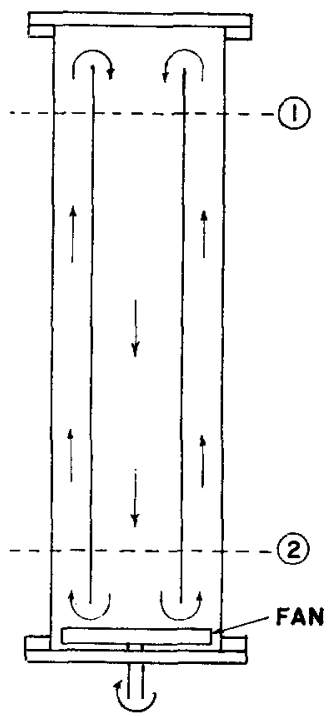




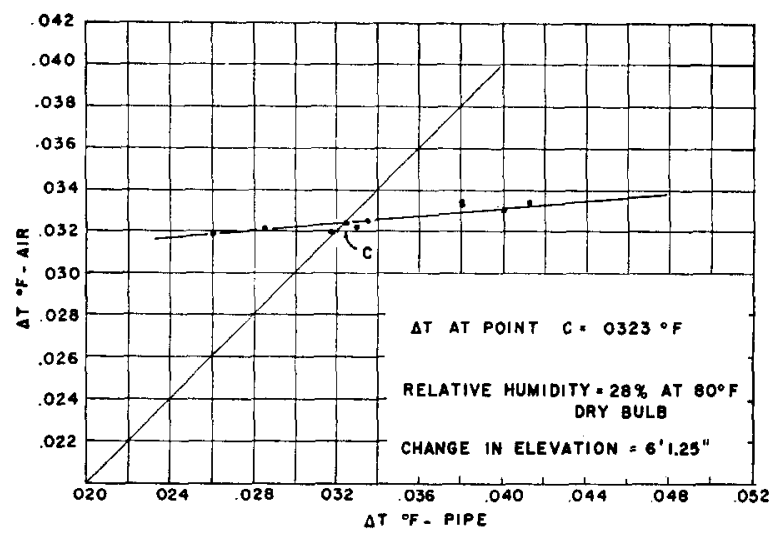

FIG. 2. Temperature increase of air in steady-flow adiabatic compression as measured on a platinum resistance thermometer.

then maintained at a constant temperature just above the room temperature, and an amount of heat equal to the energy received from the fan was transferred from the gas as it flowed up the annulus. A second platinum resistance thermometer was used in conjunction with a manual control on the heating coil to maintain the temperature constant within $\pm 0.004^{\circ} \mathrm{F}$ for several hours at a time.

2. Radial heat transfer from the gas in the annulus to the gas in the center tube was reduced by providing a one-inch thickness of Santocel insulation between the two gas streams. Calculations showed that for a gas of low thermal conductivity this heat transfer would be insignificant, but that for a gas of high thermal conductivity, the radial heat transfer would be significant. This was confirmed by the test results.

3. The matter of longitudinal temperature gradient on the wall of the inner tube was also found to be important. If the gas is to flow adiabatically down the center tube, the tube wall temperature must be equal to the gas temperature at each elevation, and thus the tube wall would have the same gradient as the change in gas temperature. Differential thermocouples were installed on the inner tube and the change in gas temperature was correlated with this longitudinal tube temperature gradient in a manner described below.

Two gases were investigated, namely, air and helium, and all tests were conducted at ambient temperature. Air was used because it is readily available and accurate specific heat data are available. Helium was used because this is probably the most difficult gas to work with in this type of apparatus. Its high specific heat, five times that of air, means that the temperature change between top and bottom is one-fifth as large as with air, making the temperature measurements more difficult. The thermal conductivity is six times as large as for air, causing greater error due to heat transfer.

The results with air are shown in Fig. 2. The temperature difference of the air is plotted against the temperature difference of the inner tube. At point $C$, where these two temperature differences are equal, it is assumed the flow takes place adiabatically. This temperature difference, when corrected for the humidity of the air, gives a value of constant pressure specific heat at $80^{\circ} \mathrm{F}$ of

$$
c_{p}=0.2417 \mathrm{Btu} / \text { pound }{ }^{\circ} \mathrm{F} \text {. }
$$

The value given in the Keenan and Kaye Gas Tables is

$$
c_{p}=0.2399 \mathrm{Btu} / \text { pound }{ }^{\circ} \mathrm{F} \text {. }
$$

The error is 0.71 percent.

The results with helium, when plotted in a similar way, gave a specific heat which was 40 percent lower than the accepted value. This error resulted from the high thermal conductivity and the fact that the temperature change in helium was so small. Using the accepted value of specific heats, in the distance of $6 \mathrm{ft} 1 \frac{1}{4} \mathrm{in}$., the temperature change in air is $0.0327^{\circ} \mathrm{F}$ as compared to $.0063^{\circ} \mathrm{F}$ for helium. It is evident that slight errors in measuring temperature and any heat transfer cause appreciable errors in the case of helium.

In conclusion, with further development, this method would seem to offer possibilities of giving useful results, particularly with gases having low specific heats and fairly low thermal conductivity. 